\title{
Hopkins Receives NAS Award in Mathematics
}

MICHAEL J. HOPKINS of Harvard University has received the 2012 NAS Award in Mathematics from the National Academy of Sciences. He was honored "for his leading role in the development of homotopy theory, which has both reinvigorated algebraic topology as a central field in mathematics and led to the resolution of the Kervaire invariant problem for framed manifolds."

The NAS Award in Mathematics was established by the AMS in commemoration of its centennial, which was celebrated in 1988. The award is presented every four years in recognition of excellence in research in the mathematical sciences published within the preceding ten years. The award carries a cash prize of US\$5,000. Previous recipients are Robert P. Langlands (1988), Robert MacPherson (1992), Andrew J. Wiles (1996), Ingrid Daubechies (2000), Dan Virgil Voiculescu (2004), and Clifford H. Taubes (2008).

\section{The Work of Michael Hopkins}

Hopkins's first major contribution to mathematics was his proof (in joint work with Ethan Devinatz and Jeff Smith) of the nilpotence theorem: if $f: K \rightarrow K$ is a pointed map from a finite CW complex to itself that is trivial on a complex bordism, then some iterate of $f$ is stably nullhomotopic. This is a foundational result, which marked the beginning of the modern era in stable homotopy theory. Using it, Hopkins and his collaborators were able to carry out a massive program (envisioned by Jack Morava, Doug Ravenel, and others) establishing deep connections between algebraic topology and the arithmetic of formal group laws. The resulting theory (sometimes called chromatic homotopy theory) has led to powerful new insights on old problems and is presently the best available tool for understanding the large-scale structural phenomena in the stable homotopy groups of spheres.

The most recent triumph of Hopkins's career is his resolution (in joint work with Mike Hill and

Elaine Kehoe is contributing writer for the Notices. Her email address is elaine1kehoe@cox.net.

DOI: http://dx.doi.org/10.1090/noti 847
Doug Ravenel) of the Kervaire invariant problem. This problem has many formulations, but perhaps the simplest is this: given a framed $n$-manifold $M$, does there always exist a (framed) bordism from $M$ to a sphere? Hill, Hopkins, and Ravenel have shown that the answer is affirmative provided that $n \neq 2$, $6,14,30,62,126$. This problem arose originally in the work of Kervaire and Milnor in the study of exotic smooth structures and was later shown by Browder to be intimately connected with the calculation of the stable homotopy groups of spheres. Despite its central role in both geometric and algebraic topology, the Kervaire invariant question remained unanswered for almost fifty years, until it was recently addressed by Hopkins and his collaborators using an array of new insights from equivariant algebraic topology.

\section{Biographical Sketch}

Michael Hopkins was born in Alexandria, Virginia, in 1958. He received both his bachelor's degree (1979) and his Ph.D. (1984) in mathematics from Northwestern University. He also received his D.Phil. in mathematics in 1984 from the University of Oxford. He held an NSF postdoctoral fellowship at Princeton University from 1984 to 1987, then advanced from instructor to assistant professor at Princeton before taking a professorship at the University of Chicago in 1988. He moved to the Massachusetts Institute of Technology in 1989 and has been professor at Harvard since 2005. He has been the recipient of a Rhodes Scholarship (1979-1982), a Presidential Young Investigator Award (1987-1995), and an Alfred P. Sloan Fellowship (1987-1992). He was awarded the Veblen Prize in Geometry in 2001. He is an associate editor of the Journal of the American Mathematical Society and of several other mathematics journals. He is a member of the American Academy of Arts and Sciences and a foreign member of the Royal Danish Academy of Sciences and Letters. 